EXTENDED REPORT

\title{
Orbital socket contracture: a complication of inflammatory orbital disease in patients with Wegener's granulomatosis
}

\author{
C Talar-Williams, M C Sneller, C A Langford, J A Smith, T A Cox, M R Robinson
}

Br J Ophthalmol 2005;89:493-497. doi: 10.1136/bjo.2004.050039

See end of article for authors' affiliations

Correspondence to:

Correspondence to:
Cheryl Talar-Williams, National Institute of

Allergy and Infectious

Diseases, NIH, Bethesda

$M D$, Room 11 B 13, 10

Center DR MSC 1863

Bethesda, MD 20892,

USA; ctwilliams@

niaid.nih.gov

Accepted for publication 1 September 2004
Aim: To describe the clinical characteristics of orbital socket contracture in patients with Wegener's granulomatosis (WG).

Methods: A retrospective cohort study The medical records of 256 patients with WG examined at the National Institutes of Health from 1967 to 2004 were reviewed to identify patients with orbital socket contracture. Details of the orbital disease including Hertel exophthalmometry readings, radiological findings, and results of eye examinations were recorded. Orbital socket contracture was defined as orbital inflammation with proptosis followed by the development of enophthalmos and radiographic evidence of residual fibrotic changes in the orbit. To examine for risk factors in the development of a contracted orbit, patients with orbital socket contracture were compared to patients without contracture with respect to multiple variables including history of orbital surgery, orbital disease severity, and major organ system involvement. The main outcome measures were the clinical characteristics of orbital socket contracture associated with inflammatory orbital disease in patients with WG.

Results: Inflammatory orbital disease occurred in 34 of 256 (13\%) patients and detailed clinical data on 18 patients were available and examined. Orbital socket contracture occurred during the clinical course in six patients; the features included restrictive ophthalmopathy (five), chronic orbital pain (three), and ischaemic optic nerve disease (two) resulting in blindness (no light perception) in one patient. The orbital socket contracture occurred within 3 months of treatment with immunosuppressive medications for inflammatory orbital disease in five patients and was not responsive to immunosuppressive medications. The median degree of enophthalmos in the contracted orbit compared with the fellow eye was $2.8 \mathrm{~mm}$ (range 1.5-3.5 mm) by Hertel exophthalmometry. There were no risk factors that predicted development of orbital socket contracture.

Conclusions: In six patients with WG and active inflammatory orbital disease, orbital socket contracture occurred during the treatment course with systemic immunosuppressive medications. The orbital socket contracture, presumably caused by orbital fibrosis, led to enophthalmos, restrictive ophthalmopathy, chronic orbital pain, and optic nerve disease and was not responsive to immunosuppressive therapy. Orbital socket contracture has not been previously reported as a complication of inflammatory orbital disease associated with WG and was an important cause of visual morbidity in our cohort of patients.
W egener's granulomatosis (WG) is a systemic vasculitis syndrome characterised by necrotising granulomatous inflammation and vasculitis of the upper and lower respiratory tracts and focal necrotising glomerulonephritis. ${ }^{1}$ Ocular involvement is common and can occur in $52-61 \%$ patients during the course of the disease. ${ }^{23}$ Ophthalmic manifestations include inflammatory orbital disease, peripheral ulcerative keratitis, episcleritis, scleritis, uveitis, nasolacrimal duct obstruction, and ischaemic optic nerve disease. ${ }^{4}$ Inflammatory orbital disease has been reported in 15-45\% of patients with WG, with associated optic nerve complications being the most common cause of vision loss. ${ }^{256}$ The most common presentation of orbital disease involves a subacute prodromal phase with escalating pain and proptosis evolving over $1-2$ months followed by a brief acceleration phase when optic nerve compression and vision loss occur. ${ }^{7}$ Early diagnosis and systemic immunosuppression can successfully treat orbital inflammation and reduce visual complications. ${ }^{25}$ In the vasculitis clinic at the National Institute of Allergy and Infectious Diseases (NIAID), a patient with WG was observed to develop significant visual morbidity associated with orbital socket contracture during the course of therapy for inflammatory orbital disease. This finding has not been previously reported in the literature. To investigate further this important observation, we performed a retrospective review of WG patients treated by the NIAID vasculitis programme over the past 37 years to evaluate the clinical features of patients who developed orbital socket contracture as a complication of inflammatory orbital disease.

\section{METHODS}

A retrospective chart review was performed on 256 patients with WG examined at the National Institutes of Health (NIH) Bethesda, MD, USA, from 1967 to 2004 to identify patients with orbital socket contracture. All study protocols were approved by the institutional review board and clinical director of the NIAID and the director of the NIH Clinical Center. All patients provided written informed consent. Data recorded included patient age at the date of WG and orbital disease diagnosis, race, sex, Hertel exophthalmometry readings, radiological findings, ocular symptoms, results of the eye examination, history of ocular and systemic manifestations of WG, cytoplasmic pattern antineutrophil cytoplasmic

Abbreviations: cANCA, cytoplasmic pattern antineutrophil cytoplasmic antibody; NAION, non-arteritic ischaemic optic neuropathy; NIAID, National Institute of Allergy and Infectious Diseases; NIH, National Institutes of Health; NLP, no light perception; WG, Wegener's granulomatosis 
antibody (cANCA) titres, and the immunosuppressive medication regimen.

The diagnosis of WG required typical clinical manifestations plus histological documentation of vasculitis or granulomatous inflammation in the absence of infection.

Inflammatory orbital disease was defined as a granulomatous inflammation involving the orbital structures complicated by oedema. This inflammatory process may develop in the extraconal or intraconal orbital space and involve the optic nerve, optic vessels, and the extraocular muscles. It is frequently associated with proptosis, and may result in visual loss from optic nerve compression and diplopia from involvement of the extraocular muscles and/or their nerves in the orbital apex or more distally. Orbital socket contracture was defined as orbital inflammation with proptosis followed by the development of enophthalmos and radiographic evidence of residual fibrotic changes in the orbit.

Possible risk factors for the development of orbital socket contracture were examined and included patient age at the time of WG and orbital disease diagnosis, race, sex, history of orbital surgery (orbital biopsy and orbital decompression), orbital disease severity (diffuse disease with $\geqslant 75 \%$ of orbit involved with circumferential optic nerve involvement or focal disease with $<50 \%$ orbit involved estimated from computed tomography (CT), number of orbital relapses (none or $\geqslant 1$ ), presence or absence of a chronic orbital mass), presence of other ophthalmic manifestations of WG (nasolacrimal duct involvement, corneal involvement, episcleritis/ scleritis, ischaemic optic nerve disease), and presence or absence of other manifestations of WG which included sinus, renal, lung, nerve, and tracheal disease (subglottic stenosis). Patients with orbital socket contracture were compared to patients without contracture with respect to these risk factors.

Differences in age between the two groups at the time of WG and orbital disease diagnosis were compared using the two sample $t$ test and the Wilcoxon rank sum test. Other risk factors were compared using a two sided Fisher's exact test. No adjustment was made for interocular correlation. Associations were considered likely to be clinically significant if the $\mathrm{p}$ value was $\leqslant 0.05$. Data were analysed using SAS 8.2 (SAS Institute Inc, Cary, NC, USA).

\section{CASE REPORTS}

\section{Patient 1}

A 16 year old white man was diagnosed in 1991 with WG. Over the following year, his disease progressed to involve the left orbit, nasolacrimal ducts, kidneys, lungs, sinuses, skin, and joints. He was treated with prednisone and methotrexate, which was later switched to cyclophosphamide. The left orbital disease resolved after 2 months of therapy and no residual infiltration was present. In 1998, the patient complained of binocular diplopia and right proptosis. Examination revealed visual acuity of 20/20 in both eyes with decreased motility in the right eye (RE) primarily in upward gaze; Hertel exophthalmometry, base 103, was $19 \mathrm{~mm} \mathrm{RE}$ and $16 \mathrm{~mm}$ left eye (LE). Computed tomography (CT) showed right orbital disease extending from the right ethmoid sinus. The patient was treated with prednisone and methotrexate, later switched to cyclophosphamide. He experienced clinical improvement but with a residual mass remaining in the right orbit. In May 1999, he developed progressive RE proptosis. Despite a multiagent immunosuppressive regimen, Hertel exophthalmometry, base 101, increased to $26.5 \mathrm{~mm}$ RE by May 2000 (fig lA). Over the next 5 months, the right orbital mass began contracting with arterialisation of the conjunctival vessels, retinal vessel tortuousity, and optic disc oedema occurring in the RE (fig 1B) clinically suggestive of an orbital apex syndrome. The patient maintained 20/20 vision and normal visual fields in both eyes until August 2000 when he developed an inferior scotoma with automated perimetry and reduced visual acuity in the RE to 20/25 with no afferent pupillary defect. He had reduced ductions in all directions in the RE. Since the aetiology of the compressive optic neuropathy appeared to be a contracting fibrotic mass around the optic nerve, decompression surgery was not performed. Despite aggressive immunosuppression with intravenous cyclophosphamide and methylprednisolone pulses followed by high dose daily prednisone, he developed progressive vision and visual field loss with a 3+ afferent pupillary defect RE in September 2000. By October 2000, he had no light perception (NLP) vision in the RE. The patient complained of progressive orbital pain over the following 2 years and became narcotic dependent. In December 2002, the orbital disease extended through the superior orbital fissure into the anterior portion of the cavernous sinus and this has remained stable through to his last examination in February 2004. The right orbital socket contracture continued to progress, with Hertel exophthalmometry of 15.5 mm RE and 19 mm LE in February 2004 (fig lC and D).

\section{Patient 2}

A 29 year old white woman had a history of WG since 1989 with involvement of the sinuses, skin, and nasolacrimal ducts treated with prednisone, cyclophosphamide, intravenous
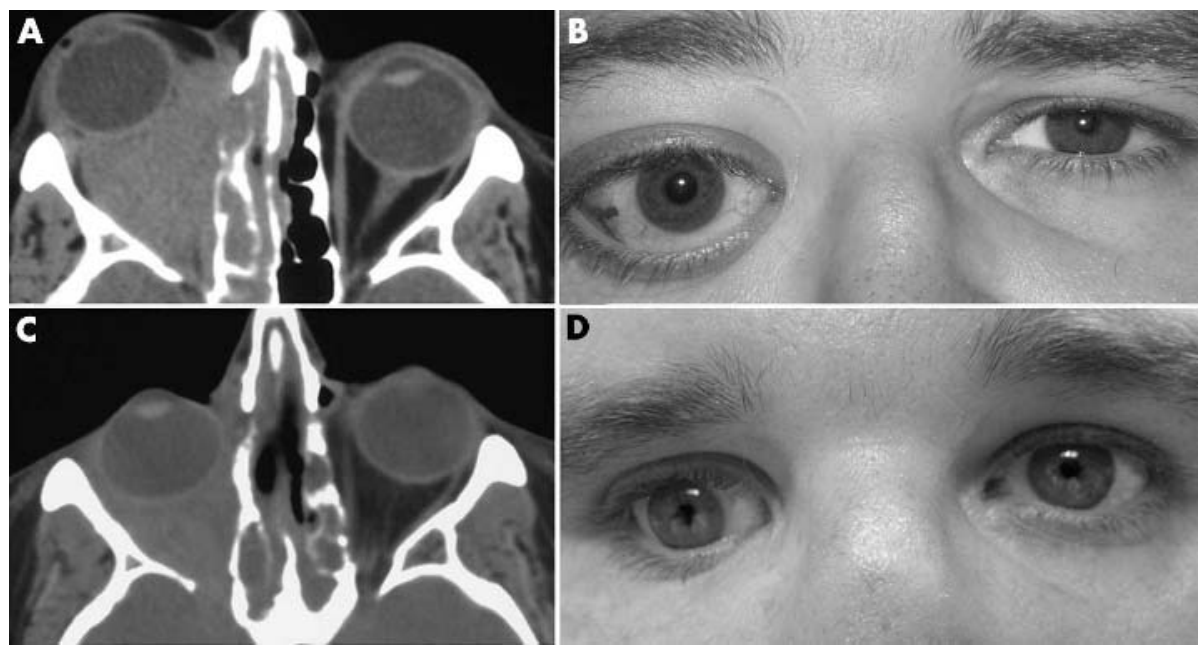

Figure 1 Case 1. Computed tomography (axial view) through the mid-orbit. (A) May 2000 showing disease progression right orbit with obliteration of normal tissue planes and increased proptosis. (B) External photograph of the patient in October 2000 showing downward displacement of the right globe and arterialisation of the conjunctival vessels. (C) February 2004 showing contraction of the right orbital mass. The orbit mass contracture resulted in optic nerve compression and deterioration of the vision to no light perception. The mass also extends through the superior orbital fissure into the anterior portion of the cavernous sinus. (D) External photograph of the patient following the orbital socket contracture showing enophthalmos. 


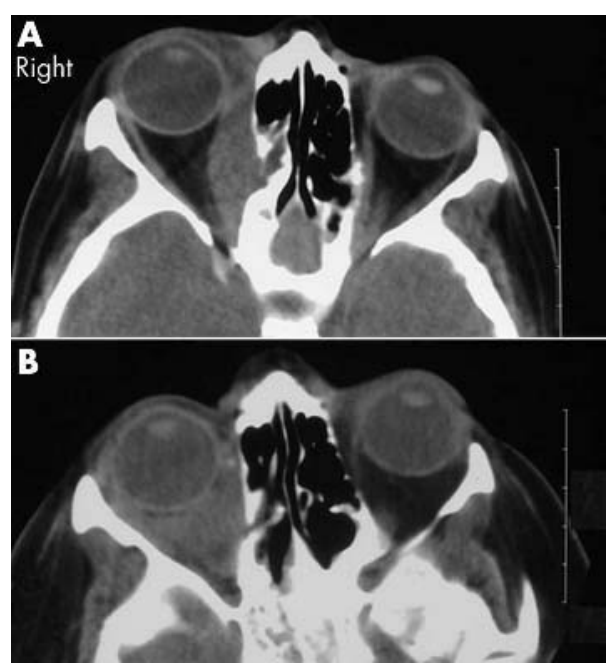

Figure 2 Case 2. (A) Computed tomography (axial view) through the mid-orbit December 1996 showing a density in the right ethmoid sinus extending into the right medial orbit with proptosis. (B) Computed tomography (axial view) through the mid-orbit November 2000 showing a diffuse right orbital opacity with contraction and enophthalmos.

immunoglobulin, and later methotrexate. She presented with right periorbital swelling in February 1996. Eye examination showed visual acuity of $20 / 16$ in both eyes and right periorbital oedema with no proptosis. CT showed right ethmoid sinus disease extending into the right medial orbit. The patient was treated with prednisone alone, continued to have progressive right orbital disease, and in May 1996, developed right proptosis (Hertel exophthalmometry, base 101, $18 \mathrm{~mm} \mathrm{RE,} 16 \mathrm{~mm} \mathrm{LE).} \mathrm{She} \mathrm{was} \mathrm{stable} \mathrm{until} \mathrm{October}$ 1996, when she complained of increased periorbital swelling. Eye examination showed normal visual acuity, normal pupillary examination, and right optic disc oedema. CT revealed an enlarging orbital mass treated with prednisone and methotrexate. Her proptosis remained stable over the following 2 months (fig 2A), and the right optic disc oedema resolved by March 1997. In August 1997, while on a tapering dose of prednisone, she developed worsening right orbital disease and optic disc oedema with no afferent pupillary defect. Her visual acuity was $20 / 20$ in both eyes and visual fields were normal. Her prednisone dose was increased to $60 \mathrm{mg}$ daily and methotrexate was restarted. Between October 1997 and December 1997, the patient complained of right orbital pain, new onset binocular diplopia, and a retracted right eye. Eye examination in December 1997 showed visual acuity of 20/20 both eyes, normal visual fields, right enophthalmos with Hertel exophthalmometry, base 100, of $14 \mathrm{~mm} \mathrm{RE},-1$ ductions in all directions, and a normal optic disc examination. Despite treatment with cyclophosphamide, over the next 3 years, she had chronic orbital pain in the contracted orbit requiring methadone for pain relief. The right enophthalmos persisted (fig $2 \mathrm{~B}$ ) and she had reduced ductions in all directions RE and normal visual acuity.

\section{Patient 3}

A 57 year old white man had a history of WG since 1967 with involvement of the right orbit, kidneys, sinuses, nerve, joints, and skin, and was being treated with prednisone in combination with methotrexate or azathioprine. In 1971, the patient developed bilateral orbital inflammatory disease LE greater than RE with disc oedema, treated with prednisone and azathioprine. Flares of his extraocular disease in the 1970s and 1980s were treated with varying medical

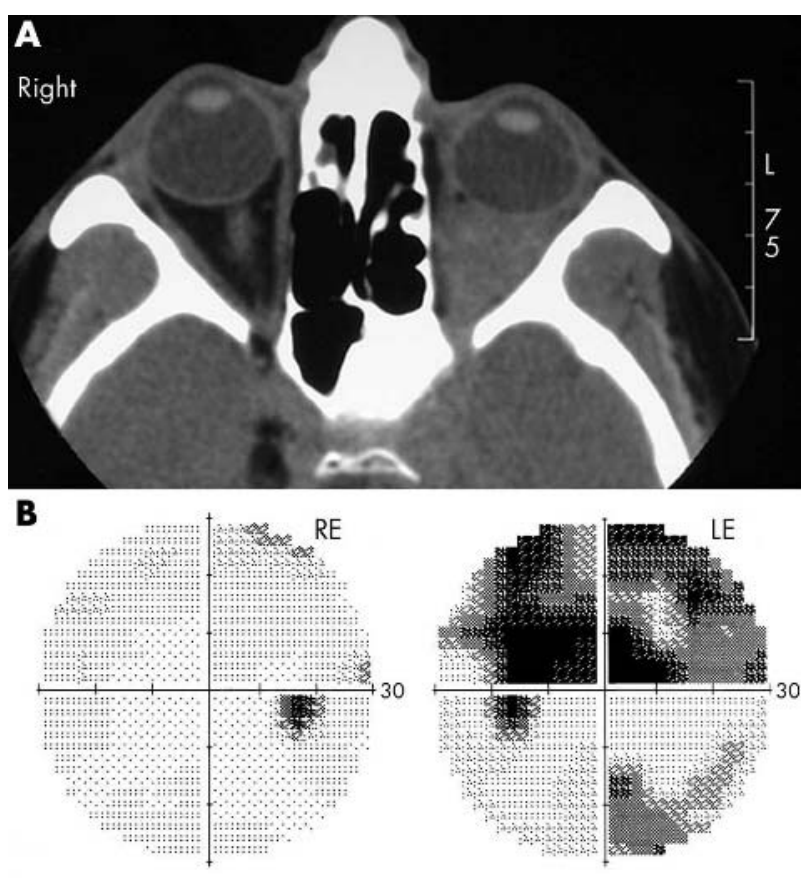

Figure 3 Case 3. (A) Computed tomography (axial view) through the mid-orbit September 2001 showing diffuse left orbital disease and a contracted socket. (B) Threshold visual fields showing a normal field RE (right eye) and a superior altitudinal field defect LE (left eye) from ischaemic optic neuropathy.

regimens including prednisone with methotrexate, azathioprine, or cyclophosphamide. A follow up eye examination in April 1990 showed visual acuity of 20/20 RE, 20/25 LE, left enophthalmos with Hertel exophthalmometry reading, base 95, $21 \mathrm{~mm} \mathrm{RE}, 17 \mathrm{~mm} \mathrm{LE}$, and bilateral optic disc pallor with no documented visual field defects. CT of the orbits in 1993 documented a contracted left orbital socket. The patient's orbital disease remained inactive. All immunosuppressive medications were discontinued in November 1998. The patient returned to the eye clinic in September 2001 complaining of blurry vision in the LE for 3 weeks. Medications included metoprolol for hypertension. The visual acuity was 20/16 RE, 20/25 LE, with Hertel exophthalmometry reading, base 96, $21.5 \mathrm{~mm} \mathrm{RE}, 18.5 \mathrm{~mm}$ LE. The eye examination showed -2 abduction and -2 adduction in the LE and no change in the optic disc pallor previously noted. CT of the orbits showed no change in the orbital anatomy (fig 3A) compared with 1993 scans. Visual fields showed a new superior altitudinal defect LE (fig 3B). The eye examination showed no relative afferent pupillary defect and bilateral optic atrophy. Since there were no signs of an inflammatory orbital flare, no clinical signs or symptoms associated with giant cell arteritis with an erythrocyte sedimentation rate of 26 , he was diagnosed with nonarteritic ischaemic optic neuropathy (NAION). Given the possibility that there was an inflammatory component involved in the pathogenesis of the ischaemia, prednisone $60 \mathrm{mg}$ daily was started then tapered off after 1 month because of no appreciable change in the visual field defect.

\section{RESULTS}

The medical records of 256 consecutive patients with Wegener's granulomatosis examined at the NIH from 1967 to 2004 were reviewed. Inflammatory orbital disease occurred in 34 of $256(13 \%)$ patients, and detailed clinical data on 18 patients were available and examined. Sixteen of the $34 \mathrm{WG}$ patients with orbital disease were not included in this study. 


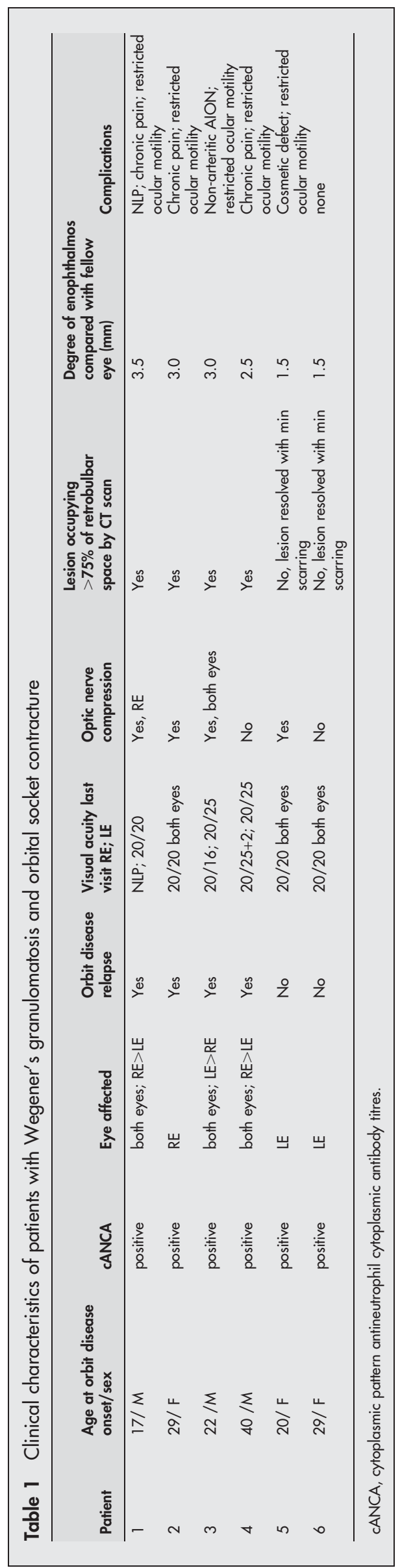

After only 2 months of follow up, one patient withdrew from protocol and one patient was excluded from the protocol because of non-compliance with follow up. Of the remaining 14 patients, serial detailed ophthalmological records, including Hertel measurements, could not be located in the medical record and many of these patients were initially seen in the 1960s and 1970s, before the development of CT scans. Orbital socket contracture occurred during the clinical course in six patients with inflammatory orbital disease. The clinical course of these patients was characterised by the development of orbital inflammatory disease with proptosis followed by the development of enophthalmos (table 1). There were three men and three women, all white. The median age at the time of diagnosis of WG and of orbital disease was 21 years (range 12-26 years) and 25.5 years (range 17-40 years), respectively. The median follow up period at the NIH was 12 years (range $4.8-22.3$ years). All patients had concurrent sinus disease. None of the patients underwent orbital decompression surgery. None of the six cases of orbital socket contracture showed radiographic evidence of a sinus contracture that may have led to decreased orbital volume and enophthalmos. One patient (patient 5) underwent a biopsy of the orbit. Patients with orbital socket contracture developed severe restriction in ocular motility (five), chronic orbital pain (three), and optic nerve disease (two) resulting in NLP vision in one eye of patient 1. The orbital socket contracture occurred within 3 months of treatment with immunosuppressive medications for active inflammatory orbital disease in five patients. In three of these patients, the orbital socket contracture occurred after a relapse of their orbital disease. The median degree of enophthalmos in the contracted orbit compared with the fellow eye was $2.8 \mathrm{~mm}$ (range 1.5-3.5 mm) by Hertel exophthalmometry at the last examination. Patients 1 and 2 developed optic nerve disease during the contracture phase, with no clinical response to an increase in immunosuppressive medications. Cytoplasmic pattern antineutrophil cytoplasmic antibody titres were positive during the clinical course in all patients; however, the changes in titres did not correlate with the orbit disease activity (data not shown). On CT scan, the orbital lesion occupied more than $75 \%$ of the retrobulbar space in four patients. In the remaining two patients, their orbital lesion resolved with minimal scarring on repeat CT scan.

In comparing patients with orbital disease who developed orbital contraction with those who did not, no significant local or systemic associations were identified with the development of orbital socket contraction. In evaluating any potential differences in the immunosuppressive medication regimens between the two groups, no trends were observed, with all patients receiving corticosteroids combined with either cyclophosphamide or methotrexate, standard medications used in the treatment of WG. ${ }^{8}$

\section{DISCUSSION}

Orbital socket contracture, most commonly reported as a consequence of metastatic orbit involvement with tumour cicatrisation or following orbital trauma, ${ }^{9}$ has not been previously reported as a complication of inflammatory orbital disease in patients with WG. The orbital socket contracture progressed despite immunosuppressive therapy, particularly in patient 1 , who was placed on an aggressive multiagent immunosuppressant regimen before losing his vision from an apparent fibrous mass contracting around the optic nerve. We suspect that orbital socket contracture was a sequela of the acute inflammatory component of the orbital disease, and that the residual fibrosis was responsible for the contracture involving the optic nerve and the associated visual morbidity. Vision loss associated with orbital WG most commonly occurs during the acute inflammatory event associated with 
proptosis, followed by optic nerve compression from the expanding orbital infiltrate, and this inflammation typically responds to systemic immunosuppressive therapy. ${ }^{25} 7$ Orbital biopsies in WG patients show a spectrum of histopathological changes including acute and chronic inflammation, fibrinoid necrosis, extensive fibrosis, and rarely vasculitis. ${ }^{21011}$ Orbital socket contracture probably represents a proliferation of fibrous tissue replacing areas of acute inflammation and necrosis, and this healing response can lead to retraction of the globe and optic nerve disease, with permanent motility restrictions. The orbital inflammatory events lead to lipolysis and the loss of orbital fat reduces the orbital volume which may contribute to the enophthalmos, especially in the two cases where there was minimal residual orbital scar. Although orbital decompression has been successful in treating optic nerve compression during the acute phase of orbital inflammation, ${ }^{12}{ }^{13}$ the role for this procedure in treating a fibrosing orbital socket contracture is not clear. Other manifestations of WG that involve fibrosis and contracture of tissues include cicatricial conjunctivitis, ${ }^{14}{ }_{15}$ nasolacrimal duct obstruction, ${ }^{16}$ and subglottic stenosis, a disease manifestation that can lead to laryngeal obstruction and respiratory failure. ${ }^{17}$ Similar to orbital socket contracture, these other fibrosing manifestations of WG are poorly responsive to immunosuppressive medications. ${ }^{18}$

The ischaemic event in the optic nerve of the contracted orbit in patient 3 occurred years after the orbital socket contracture had developed. Although arteritic anterior ischaemic optic neuropathy has been reported in patients with WG ${ }^{19}{ }^{20}$ our patient had an altitudinal field defect with preserved central vision, a normal erythrocyte sedimentation rate, and no clinical response to corticosteroids, all suggestive of a NAION. Although the patient had no clinical evidence of active orbital Wegener's granulomatosis (that is, no increase in proptosis, no lid oedema, erythema, or pain) or radiographic changes compared with previous scans, we could not rule out the possibility that the patient developed a low grade orbital flare with optic nerve compression and subsequent visual field loss. Nevertheless, this patient had risk factors for a NAION including a history of hypertension, possibly reduced blood flow to the optic disc ${ }^{21}$ from a contracted retrobulbar mass, and previous optic atrophy.

The aetiology of the chronic pain that developed in three of the patients, with two requiring chronic narcotic therapy, was not known, but compression of the sensory nerves from the orbital socket contracture was assumed.

In summary, orbital socket contracture in six patients with WG occurred during the treatment course of active inflammatory orbital disease with systemic immunosuppressive medications. The orbital socket contracture, presumably caused by orbital fibrosis, led to restrictive ophthalmopathy, chronic orbital pain, and optic nerve disease, and was not responsive to immunosuppressive therapy. Orbital socket contracture, not previously reported as a complication of inflammatory orbital disease associated with WG, was an important cause of visual morbidity in our cohort of patients.

\section{ACKNOWLEDGEMENTS}

The authors thank Kristen E McCabe, RN, the Clinical Center nursing staff at the National Eye Institute and National Institute of Allergy and Infectious Diseases for the care of our patients, and Dr Anthony S Fauci for his work in establishing the National Institute of Allergy and Infectious Diseases Vasculitis Program.

\section{Authors' affiliations}

C Talar-Williams, M C Sneller, C A Langford, National Institute of Allergy and Infectious Diseases, NIH, Bethesda, MD, USA

J A Smith, T A Cox, M R Robinson, National Eye Institute, NIH, Bethesda, MD, USA

Presented in a poster at the annual meeting of the American Academy of Ophthalmology, New Orleans, Louisiana, November 2001.

\section{REFERENCES}

1 Sneller MC. Wegener's granulomatosis. JAMA 1995;273:1288-91.

2 Hoffman GS, Kerr GS, Leavitt RY, et al. Wegener granulomatosis: an analysis of 158 patients. Ann Intern Med 1992;116:488-98.

3 Reinhold-Keller E, Beuge N, Latza U, et al. An interdisciplinary approach to the care of patients with Wegener's granulomatosis: long-term outcome in 155 patients. Arthritis Rheum 2000;43:1021-32.

4 Harman LE, Margo CE. Wegener's granulomatosis. Surv Ophthalmol 1998;42:458-80.

5 Bullen CL, Liesegang TJ, McDonald TJ, et al. Ocular complications of Wegener's granulomatosis. Ophthalmology 1983;90:279-90.

6 Woo TL, Francis IC, Wilcsek GA, et al. Australasian orbital and adnexal Wegener's granulomatosis. Ophthalmology 2001;108:1535-43.

7 Perry SR, Rootman J, White VA. The clinical and pathologic constellation of Wegener granulomatosis of the orbit. Ophthalmology 1997; 104:683-94.

8 Langford CA, Sneller MC. Update on the diagnosis and treatment of Wegener's granulomatosis. Adv Intern Med 2001;46:177-206.

9 Cline RA, Rootman J. Enophthalmos: a clinical review. Ophthalmology 1984;91:229-37.

10 Straatsma BR. Ocular manifestations of Wegener's granulomatosis. Am J Ophthalmol 1957:789-99.

11 Devaney KO, Travis WD, Hoffman G, et al. Interpretation of head and neck biopsies in Wegener's granulomatosis. A pathologic study of 126 biopsies in 70 patients. Am J Surg Pathol 1990;14:555-64.

12 Duncker G, Nolle B, Asmus R, et al. Orbital involvement in Wegener's granulomatosis. Adv Exp Med Biol 1993;336:315-17.

13 Thawley SE. Wegener's granulomatosis: unusual indication for orbital decompression. Laryngoscope 1979;89:145-54.

14 Jordan DR, Addison DJ. Wegener's granulomatosis. Eyelid and conjunctival manifestations as the presenting feature in two individuals. Ophthalmology 1994; 101:602-7

15 Meier FM, Messmer EP, Bernaver W. Wegener's granulomatosis as a cause of cicatrising conjunctivitis. [Letter] Br J Ophthalmol 2001;85:628.

16 Hardwig PW, Bartley GB, Garrity JA. Surgical management of nasolacrimal duct obstruction in patients with Wegener's granulomatosis. Ophthalmology 1992;99:133-9.

17 Matt BH. Wegener's granulomatosis, acute laryngotracheal airway obstruction and death in a 17-year-old female: case report and review of the literature. Int J Pediatr Otorhinolaryngol 1996;37: 163-72.

18 Langford CA, Sneller MC, Hallahan CW, et al. Clinical features and therapeutic management of subglottic stenosis in patients with Wegener's granulomatosis. Arthritis Rheum 1996;39:1754-60.

19 Belden CJ, Hamed LM, Mancuso AA. Bilateral isolated retrobulbar optic neuropathy in limited Wegener's granulomatosis. J Clin Neuroophthalmol 1993;13:119-23.

20 Paul B, McElvanney AM, Agarwal S, et al. Two rare causes of posterior ischaemic optic neuropathy: eosinophilic fasciitis and Wegener's granulomatosis. Br J Ophthalmol 2002;86:1066-8.

21 Hayreh SS. Factors influencing blood flow in the optic nerve head. J Glaucoma 1997;6:412-25. 